\title{
Article \\ Deactivation of Zeolite Catalysts in the Prins Reaction between Propene and Formaldehyde in the Liquid Phase
}

\author{
Stanislav P. Bedenko * (1), Konstantin I. Dement'ev (1) and Valentin F. Tret'yakov ${ }^{\dagger}$ \\ A.V. Topchiev Institute of Petrochemical Synthesis, Russian Academy of Sciences (TIPS RAS), \\ Leninsky Prospect 29, 119991 Moscow, Russia; kdementev@ips.ac.ru (K.I.D.); tretyakov@ips.ac.ru (V.F.T.) \\ * Correspondence: bedenko@ips.ac.ru \\ † Deceased.
}

check for updates

Citation: Bedenko, S.P.; Dement'ev, K.I.; Tret'yakov, V.F. Deactivation of Zeolite Catalysts in the Prins Reaction between Propene and Formaldehyde in the Liquid Phase. Catalysts 2021, 11, 1181. https://doi.org/10.3390/ catal11101181

Academic Editor: Arnaud Travert

Received: 2 September 2021

Accepted: 27 September 2021

Published: 28 September 2021

Publisher's Note: MDPI stays neutral with regard to jurisdictional claims in published maps and institutional affiliations.

Copyright: (C) 2021 by the authors. Licensee MDPI, Basel, Switzerland. This article is an open access article distributed under the terms and conditions of the Creative Commons Attribution (CC BY) license (https:// creativecommons.org/licenses/by/ $4.0 /)$

\begin{abstract}
The Prins reaction between propene and formaldehyde was studied over H-BEA, H-FAU, $\mathrm{H}-\mathrm{MFI}$ and $\mathrm{H}-\mathrm{MOR}$ zeolites at $150^{\circ} \mathrm{C}$ in liquid phase. It was found that the $\mathrm{H}$-BEA sample is the most active and selective toward buta-1,3-diene; the H-MFI is a potential catalyst for 3-buten-1-ol synthesis, while H-FAU can be used for 4-methyl-1,3-dioxane production. It had been confirmed that zeolite textural and acidic properties influence catalyst behaviour: the acidic properties influence sample activity, while product distribution is controlled by pore volume and effective pore diameter. The sample's deactivation process had been studied and the kinetic model of deactivation was proposed It was shown that the deactivation rate for the H-MFI catalyst is four times greater than for the H-BEA catalyst, probably because its strong/weak acid sites ratio is much more high than for the H-BEA.
\end{abstract}

Keywords: zeolite catalysts; Prins reaction; propene; formaldehyde; 3-buten-1-ol; buta-1,3-diene; catalyst deactivation

\section{Introduction}

Buta-1,3-diene is now one of the most important petrochemical products. Its annual worldwide production is approximately 14.5 million tons and growing [1]. Butadiene is used mainly for the production of synthetic rubber and some other polymers (such as ABS plastic) [2]. The naphtha stream cracking, which is a basic industrial process for buta-1,3-diene production has a relatively low buta-1,3-diene yield [3-5] and is unable to meet the growing market demand [3]. In addition, steam cracking does not meet criteria for sustainable production, since it uses fossil hydrocarbons as a feed, so new technologies for "green" butadiene production should be developed.

The Prins reaction looks like a promising approach for target industrial production of various diene hydrocarbons. It can use bio-based olefins as a feed, obtained from bioderived syngas and carbon dioxide through methanol and dimethyl, ether via MTO and DTO processes; aldehyde component of the reaction also can be produced from methanol or extracted from pyrolysis bio-oils [4-7]. However, current industrial realizations of Prins reaction have some serious ecological drawbacks because of its use of $\mathrm{H}_{2} \mathrm{SO}_{4}$ and $\mathrm{H}_{3} \mathrm{PO}_{4}$ as a catalyst. In recent years, it has been shown that heterogeneous acidic materials, especially zeolites, can be used as catalysts in the Prins reaction for the production of dienes and unsaturated alcohols [8-13].

Investigation of the propene-formaldehyde Prins reaction, catalysed by Zn-modified MFI, FER and BEA zeolites, had been carried out by Vasiliadou et al. [14]. It was pointed out, that Zn-MFI is a promising catalyst for 3-buten-1-ol synthesis and Zn-BEA can be used for buta-1,3-diene production. Zn-FER was inactive in the reaction due to rapid deactivation. Theoretical studies [15-17] have showed, that FAU zeolites also can be used as a catalyst in Prins reaction, however, activity of zeolites of this type is not experimentally studied to date. Among other zeolites, H-MOR was used for 2-methylbuta-1,3-diene synthesis catalyst [18], but it was never used for buta-1,3-diene synthesis. 
Deactivation of zeolite catalysts has a great influence on their catalytic performance. It was shown [14] that total acidity is one of the main factors that determines the deactivation rate of zeolite catalysts in the propene-formaldehyde Prins reaction; spent H-MFI catalysts with lower $\mathrm{Si} / \mathrm{Al}$ ratio were found to be deactivated by high molecular weight products (coke), while $\mathrm{H}-\mathrm{MFI}$ catalysts with high $\mathrm{Si} / \mathrm{Al}$ ratio were deactivated by strongly absorbed molecules of solvent water and reaction products. Since deactivation by coke is a big drawback for the zeolite catalysts and often leads to significant changes in catalyst behaviour, this problem has attracted much attention [19-22]; however, deactivation of the zeolite catalysts in the Prins reaction has not yet been studied. Recently we showed that zeolite topology has a great influence on catalyst activity and selectivity in the Prins reaction between 2-methylpropene and formaldehyde [23]. The aim of the present work is to explore the propylene-formaldehyde Prins reaction over H-BEA, H-FAU, H-MFI and H-MOR zeolites to estimate the influence of zeolite structure on activity and selectivity, and to study the deactivation patterns of the most promising samples.

\section{Results and Discussion}

\subsection{Catalysts Characterization}

Textural and acidic properties of the catalysts are represented in Table 1. The textural properties of the zeolite samples are consistent with their structure; specific surface (S), micropore volume $\left(\mathrm{V}_{\mathrm{MP}}\right)$ and efficient pore diameter $\left(\mathrm{D}_{\mathrm{P}}\right)$ increased in the order $\mathrm{H}-\mathrm{MFI}<\mathrm{H}-\mathrm{MOR}$ $<\mathrm{H}$-BEA $<\mathrm{H}-\mathrm{FAU}$. Total acidity increased in the order H-BEA $<\mathrm{H}-\mathrm{FAU}<\mathrm{H}-\mathrm{MFI}<\mathrm{H}-\mathrm{MOR}$, while strong/weak site ratio (S/W ratio) declined in the following order: H-MFI > H-FAU $>\mathrm{H}-\mathrm{MOR}>\mathrm{H}-\mathrm{BEA}$. It should be noted that weak acid sites are dominant for H-BEA and H-MOR, while H-MFI and H-FAU had a significant amount of the strong acid sites.

Table 1. Textural and acidic properties of samples.

\begin{tabular}{|c|c|c|c|c|c|c|}
\hline Sample & Si/Al & $\mathrm{S}, \mathrm{m}^{2} / \mathrm{g}$ & $\mathrm{V}_{\mathrm{MP}}, \mathrm{cm}^{3} / \mathrm{g}$ & $\mathrm{D}_{\mathbf{P}}, \AA$ & Acid Site Number, $\mu \mathrm{mol} / \mathrm{g}$ & S/W Ratio \\
\hline H-BEA & 12.5 & 540 & 0.22 & 7.2 & 700 & 0.25 \\
\hline H-FAU & 15 & 720 & 0.26 & 10.3 & 390 & 0.55 \\
\hline H-MFI & 15 & 360 & 0.18 & 5.6 & 1120 & 0.60 \\
\hline H-MOR & 10 & 480 & 0.19 & 5.7 & 1450 & 0.38 \\
\hline
\end{tabular}

\subsection{Catalytic Tests}

Figure 1 demonstrate the reaction network for the propene-formaldehyde Prins condensation. According to $[8,13,14,23]$, the primary product of the olefin-formaldehyde condensation is $\gamma$-unsaturated alcohol, which undergoes further transformations. The main reaction route here is dehydration, which leads to diene hydrocarbon and a condensation with formaldehyde to form 1.3-dioxanes. Diols, for example, butane-1,3-diol, are not produced, due to anhydrous reaction medium (1,4-dioxane). The 4-methyl-1,3-dioxane was not considered as the buta-1,3-diene precursor here since it is an inert chemical in these reaction conditions, however, it can probably be used for buta-1,3-diene production, for example, 4,4-dimethyl-1,3-dioxane is an industrial chemical used for isoprene production [2]. So, target products for production of butadiene are the only 3-buten-1-ol. The results of catalytic tests with various catalysts are shown in Table 2. According to obtained data, the H-BEA was the most active and selective catalyst toward buta-1,3-diene, but selectivity toward 3,6-dihydro-2H-pyran and tetrahydro-2H-pyran-4-ol is also high. The H-BEA sample can be considered as a very promising catalyst to buta-1,3-diene synthesis, although it is necessary to limit side reactions, in particular buta-1,3-diene condensation with formaldehyde. 


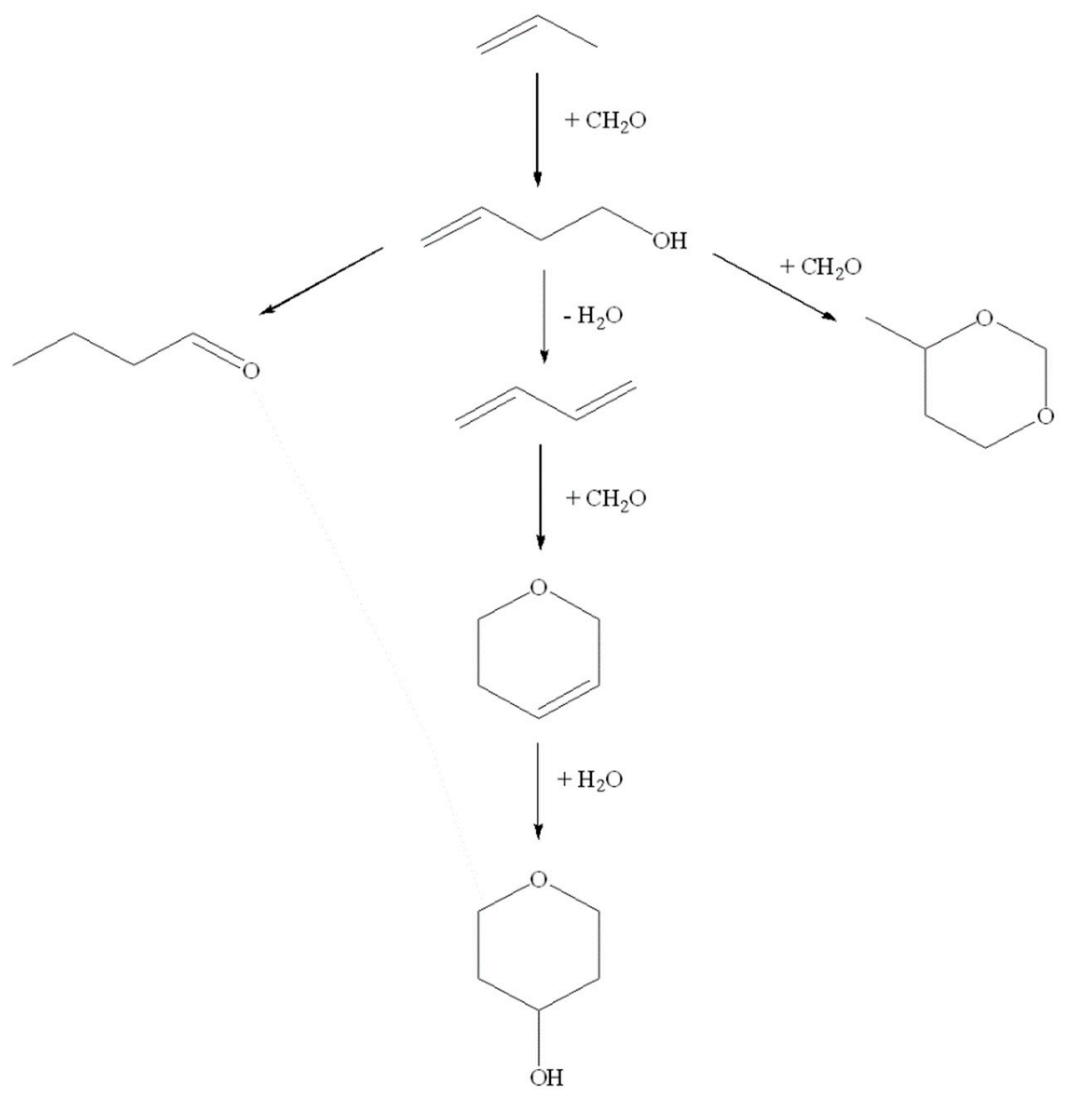

Figure 1. The main reaction network for propylene-formaldehyde condensation.

Table 2. Formaldehyde conversion $(\mathrm{X})$ and product selectivity $\left(2 \mathrm{~h}, 150{ }^{\circ} \mathrm{C}\right)$.

\begin{tabular}{|c|c|c|c|c|c|c|c|c|}
\hline \multirow[b]{2}{*}{ Sample } & \multirow[b]{2}{*}{$X, \%$} & \multicolumn{7}{|c|}{ Selectivity, \% } \\
\hline & & & & & & & & Other \\
\hline H-BEA & 45.0 & 1.8 & 1.0 & 55.6 & 19.1 & 9.4 & 10.0 & 3.1 \\
\hline H-FAU & 41.2 & 3.6 & 1.2 & 43.5 & 32.5 & 6.5 & 9.2 & 3.5 \\
\hline H-MFI & 19.9 & 33.8 & 14.8 & 28.0 & 7.8 & 8.9 & 4.8 & 1.9 \\
\hline H-MOR & 14.0 & 19.3 & 10.5 & 34.9 & 17.3 & 9.0 & 6.4 & 2.6 \\
\hline H-BEA ${ }^{2}$ & 19.4 & 4.2 & 1.6 & 59.1 & 21.4 & 6.5 & 5.8 & 1.4 \\
\hline
\end{tabular}

${ }^{1}$ A sum of a minor reaction products, such as 2-buten-1-ol, methyl formate, et al. ${ }^{2}$ After $0.75 \mathrm{~h}$ reaction.

The H-FAU catalyst showed activity similar to H-BEA and selectivity toward buta1,3-diene. As in the previous case, the H-FAU produced many side products (summary selectivity about $50 \%$ ), with low selectivity toward buta-1,3-diene precursors (a summary about $15 \%$ ); however about $33 \%$ of converted formaldehyde was spent on 4-methyl-1,3dioxane production, thus the H-FAU was concluded to be more suitable for its synthesis and can be used for a two-stage process if its modifications will lead to selectivity growth

The H-MFI and H-MOR catalysts have twice less activity compared with the H-FAU and H-BEA catalysts. The 3-buten-1-ol was the main reaction product over the H-MFI, while selectivity toward buta-1,3-diene was the lowest among all the other catalysts. At the same time, the H-MFI had the least selectivity toward side products (less than $30 \%$ ) and 4-methyl-1,3-dioxane. H-MFI catalyst probably is the most appropriate catalyst for 3-buten1-ol production. Selectivity toward 3-buten-1-ol and buta-1,3-diene for the H-MOR catalyst is lower compared with the H-MFI, but higher, than for H-BEA and H-FAU, with summary 
selectivity toward side products about $45 \%$. This sample was concluded as unsuitable for the propylene-formaldehyde Prins reaction due to its poor activity and selectivity.

The comparison of H-BEA and H-MFI behaviour at the equitable conversion level (about 20\%) emphasized that differences in selectivity of the samples were due to different properties rather than reaction kinetics. We propose that topology and acidity are the main factors that determine activity and selectivity of the zeolite catalysts in the Prins reaction. Recently, we published our study about the Prins reaction between 2-methylpropene and formaldehyde over H-BEA and H-MFI zeolites [23]: the H-BEA was pointed as the promising catalyst for 2-methylbuta-1,3-diene synthesis, while H-MFI was very selective toward 3-methylbut-3-eneol-1. The catalyst samples studied in the present work for propyleneformaldehyde condensation follow the same tendencies; however, the performance of the zeolites was not the same; the activity of samples in the 2-methylpropene-formaldehyde case was twice that in the propene-formaldehyde reaction, which is consistent with existing literature data about olefins reactivity in the Prins reaction [24]. As is already known, the Prins reaction proceeds via the carbocationic mechanism and intermediate stabilization can limit the reaction rate; the 1-hydroxy-3-methylbutan-2-ylium carbocation, that is the 2-methylpropene-formaldehyde reaction intermediate, is more stable than 1-hydroxybutan2-ylim carbocation, produced via the propene-formaldehyde reaction.

Apparently, catalyst performance is controlled by zeolite pore topology and acidic properties; large 12-T-membered pores (BEA, FAU and 12-T MOR) and medium 10-Tmembered MFI pores cannot prevent the formation of bulky products (such as 3,6-dihydro2H-pyran or tetrahydro-2H-pyran-4-ol), while small 8-T MOR pores are rapidly deactivated by coke. The H-MFI is the most suitable for 3-buten-1-ol synthesis among the studied samples, however, it should be stated that modification of H-MFI is required for selective 3-buten-1-ol synthesis, or it is preferred to use another 10-T-membered pore samples with fewer width than H-MFI has. Acid properties also make a great contribution to catalyst performance; the most active samples (H-BEA and H-FAU) had fewer acid sites numbers and had a broad product distribution, while H-MFI and H-MOR were more selective toward 3-buten-1-ol and had a poor conversion of formaldehyde. According to the results and previous reports $[13,14,23]$, we assumed that samples with a higher total acid are less active due to faster deactivation of the sites by coke.

\subsection{Catalytic Deactivation}

To study a deactivation pattern for catalysts TGA/DSC/DTA, an analysis of spend samples was carried out (Figure S5). All samples contain physiosorbed substances, such as reagents, reaction products (including water), solvent and coke. The samples with large pores, such as H-BEA and H-FAU, had a significant amount of coke (22.0 wt.\% and 22.2 wt. $\%$, respectively), while H-MFI and H-MOR had much less coke in structure (10.3 wt.\% and $15.1 \mathrm{wt} . \%$, respectively). Normalized thermal effects $\mathrm{Q}_{\mathrm{I}}$ and $\mathrm{Q}_{\text {II }}$ have been calculated from DSC curves (Figure S6) and are shown in Table 3; $\mathrm{Q}_{\mathrm{I}}$ was associated with desorption of physiosorbed solvent, reactants and products, while $\mathrm{Q}_{\text {II }}$ clearly belongs to coke oxidation with heat emission.

Table 3. Weight changing and normalized thermal effects of spend samples $\left(2 \mathrm{~h}, 150^{\circ} \mathrm{C}\right)$.

\begin{tabular}{ccccc}
\hline Sample & $\Delta \mathbf{m}_{\mathbf{I}}, \mathbf{w t} . \%$ & $\Delta \mathbf{m}_{\mathbf{I I}}, \mathbf{w t} . \%$ & $\mathbf{Q}_{\mathbf{I}}, \mathbf{J} / \mathbf{g}$ & $\mathbf{Q}_{\mathbf{I I}}, \mathbf{J} / \mathbf{g}$ \\
\hline H-BEA & 5.0 & 22.0 & -37.5 & 1235.9 \\
H-FAU & 6.3 & 22.2 & -33.3 & 3127.2 \\
H-MFI & 6.3 & 10.3 & -140.6 & 712.7 \\
H-MOR & 5.9 & 15.1 & -106.1 & 740.3 \\
\hline
\end{tabular}

According to obtained results, there is a correlation between the thermal effects/weight of coke and samples total pore volume. Moreover, other textural properties, such as efficient pore width, affect selectivity of the sample, thus we assumed that textural properties control sample deactivation. There are also differences in coke combustion temperatures 
for catalyst samples, which can be attributed to the various molecular weight of the coke. For the H-MOR catalyst, average molecular weight of the coke is least among the other catalysts since an exothermal effect has been observed in the region $300-400{ }^{\circ} \mathrm{C}$. Both wide pore catalyst samples (H-BEA and H-FAU) showed a broad peak at the DSC curves in the high-temperature region that confirmed the formation of coke with various molecular weights. The peak at the H-MFI curve was wider than H-MOR one, but narrower than H-BEA or H-FAU, thus H-MFI coke probably had median molecular weight [25].

In addition, stability tests of H-MFI and H-BEA were carried out. These samples were chosen due to their catalytic behaviour (activity, high selectivity toward buta-1,3-diene or toward 3-buten-1-ol). For this aim, spend samples had been collected after the reaction, then filtered, dried at $110^{\circ} \mathrm{C}$, and recycled without calcination. The obtained results are shown in Figure 2a,b.

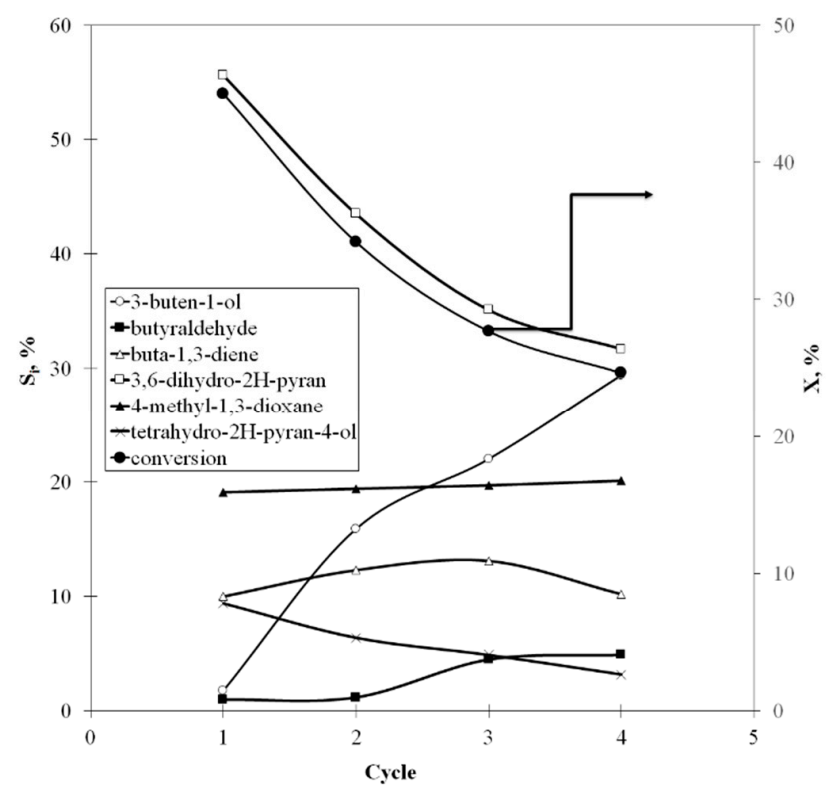

(a)

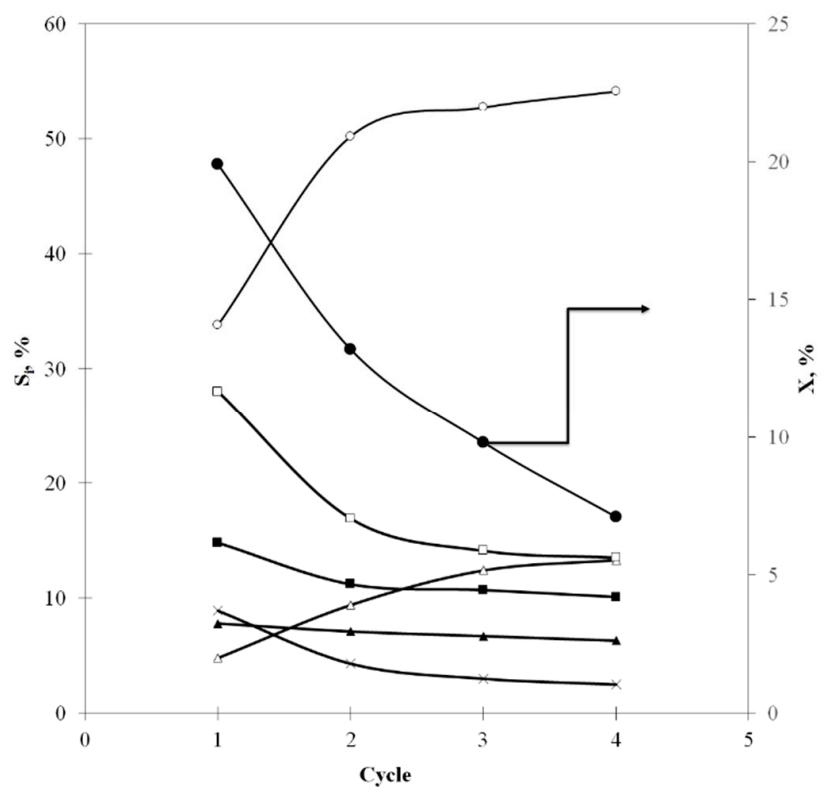

(b)

Figure 2. Formaldehyde conversion and product selectivity during stability test over H-BEA (a) and H-MFI (b).

It is obvious that the conversion of formaldehyde fell down cycle by cycle, at the same time, selectivity toward undesired bulky products also decreased over both samples. This behaviour clearly emphasized that acid properties of the sample and pore topology influence on activity and selectivity of the samples; zeolites with high values of accessible acidic sites had high activity and poor selectivity toward 3-buten-1-ol and buta-1,3-diene; coke can partially or fully block pores and change the accessibility of some sites which leads to a decrease in conversion and an increase in 3-buten-1-ol and buta-1,3-diene selectivity. Moreover, acid site strength also influences catalytic behaviour and deactivation of the catalyst: activity of H-BEA catalyst declines slower with reaction time compared with the H-MFI. Activity of the spent samples can be completely restored by calcination overnight (Table S2).

Based on obtained results, deactivation rate and initial activity (A) for both catalysts were estimated. Obtained curves are shown in Supporting Information (Figures S7 and S8) and on Figure 3. In both cases, H-BEA (Figure 3a) and H-MFI (Figure 3b) curves were a linear on $\mathrm{A}^{-1}$ vs. $t_{R}$ plots, which means, that the deactivation of H-MFI and H-BEA catalysts in the Prins condensation is of second order. According to [26], the secondorder process is due to kinetics phenomena, where two sites are blocked via the one cycle of deactivation. Thus, we proposed, that the sites are selectively deactivated by bulky molecules (such as 3,6-dihydro-2H-pyran or its transformation products), while the pore 
mouth does not block by coke. The corresponding kinetic equation and kinetic parameters are shown in Table 4. According to obtained data, the deactivation of H-BEA and H-MFI have different rates: H-BEA deactivation rate is lower than the H-MFI has, because H-MFI deactivation constant $\mathrm{k}$ was four time greater than H-BEA one. Probably, the acid sites distribution is mandatory for the deactivation rate of catalysts; the more the strong/weak acid sites ratio, the faster the deactivation of the catalysts. It is known that strong acid sites deactivate faster than weak ones [19], while textural properties control selectivity and accessibility of the sites [27]. Obtained models for catalyst deactivation are shown in Supporting Information (Figures S9 and S10).

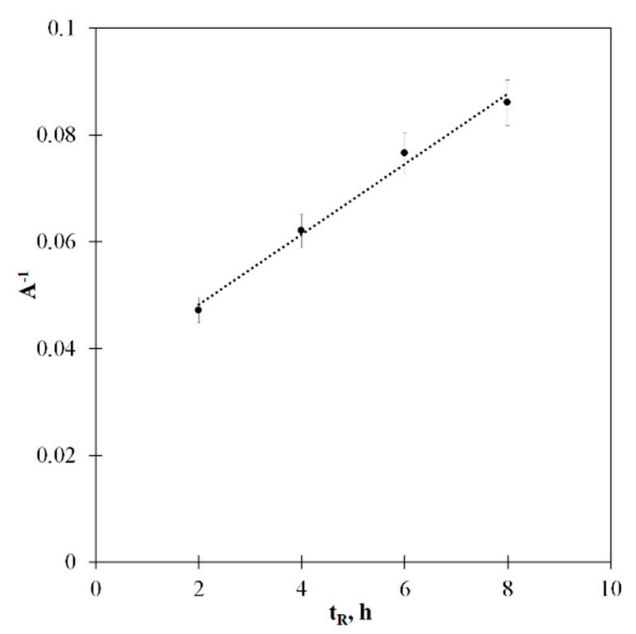

(a)

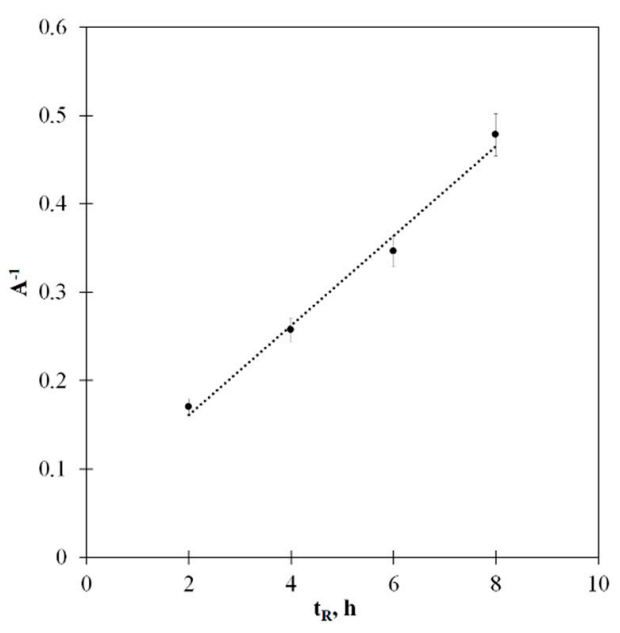

(b)

Figure 3. The H-BEA (a) and H-MFI (b) deactivation curves in $A^{-1}$ vs. $t_{R}$ scale.

Table 4. Model of H-BEA and H-MFI deactivation at $150{ }^{\circ} \mathrm{C}$.

\begin{tabular}{ccc}
\hline Sample & $\mathbf{k}, \mathbf{1 0}^{\mathbf{- 1}} \mathbf{h}^{-\mathbf{1}}$ & $\mathbf{A}^{\mathbf{0}}$ \\
\hline H-BEA & $1.90 \pm 0.05$ & $28.5 \pm 0.3$ \\
H-MFI & $8.40 \pm 0.10$ & $16.6 \pm 0.2$ \\
\hline
\end{tabular}

\section{Materials and Methods}

\subsection{Catalysts and Materials}

The propene-formaldehyde Prins reaction was investigated over zeolites of BEA, FAU, MFI and MOR types, supplied by Zeolyst International (CP814E, CBV 720, CBV 3024E and CBV 21A, respectively). The protonic form of all zeolites was obtained by calcination in air at $600{ }^{\circ} \mathrm{C}$ overnight. All chemicals were purchased from commercial sources and used without further purification: propene (99.6\%, Voessen, Moscow, Russia), paraformaldehyde (extra pure, Acros Organics, New Jersey, NJ, USA) and 1,4-dioxane (extra pure, Acros Organics, New Jersey, NJ, USA). Obtained samples were analysed by XRD (Rigaku Rotaflex RU-200, CuK $\alpha$ radiation) and XRF analysis (ARL Perform'x Sequential XFR with $2.5 \mathrm{~kW}$ power) to estimate phase and elemental composition. XRD patterns and elemental composition are shown on Figure S1 and Table S1 in Supporting Information.

\subsection{Catalyst Characterization}

Textural properties of the catalysts were studied by low-temperature nitrogen adsorption using ASAP-2020 analyser (Micromeritics, Norcross, GA, USA). Specific area (S) and micropore volume $\left(\mathrm{V}_{\mathrm{MP}}\right)$ had been obtained from low-temperature nitrogen physisorption isotherms (Figure S2) by $t$-plot method, while effective pore diameter $\left(D_{P}\right)$ was estimated by Horwath-Kawazoe method from differential pore volume curves (Figure S3). 
Total acidity of the catalysts were obtained using TPD-NH $\mathrm{N}_{3}$ method using USGA-101 equipped with a TCD; the samples were placed into a quartz reactor, heated under He flow to $500{ }^{\circ} \mathrm{C}$, calcined at this temperature for $1 \mathrm{~h}$ and finally cooled to $60{ }^{\circ} \mathrm{C}$. Ammonia adsorption was carried out using a dry $\mathrm{NH}_{3} / \mathrm{N}_{2}$ gas mixture (1/9) for $15 \mathrm{~min}$, and then physically adsorbed $\mathrm{NH}_{3}$ was removed at $100{ }^{\circ} \mathrm{C}$ in a He flow for $1 \mathrm{~h}$, after which the temperature in the reactor was linearly increased to $600{ }^{\circ} \mathrm{C}$ (rate $8{ }^{\circ} \mathrm{C} / \mathrm{min}$ ). TPD curves are shown on Figure S4; the peaks with max at $200-300^{\circ} \mathrm{C}$ were attributed to the weak acid sites, while the ones with max at $350-450{ }^{\circ} \mathrm{C}$ were assigned to the strong acid sites.

The coke content for the spent catalysts was measured by a Mettler Toledo TGA/DSC3+ thermogravimetric analyser. The spent catalysts were heated in $150 \mu \mathrm{L}$ alumina crucible from 30 to $1000{ }^{\circ} \mathrm{C}$ under air flow $(50 \mathrm{~mL} / \mathrm{min})$ with a heating rate of $10^{\circ} \mathrm{C} / \mathrm{min}$. TGA and DSC curves are shown on Figures S5 and S6, respectively.

\subsection{Catalytic Tests}

The propene-formaldehyde Prins reaction was carried out in a $50 \mathrm{~mL}$ stainless steel autoclave reactor with intensive stirring at $600 \mathrm{rpm}$. The reaction temperature was controlled by an electrical oven with a controller. In a typical experiment, the reactor was loaded by $1.0 \mathrm{~g}$ of paraformaldehyde, $6.0 \mathrm{~g}$ of propene, $0.25 \mathrm{~g}$ of catalyst and $20.0 \mathrm{~mL}$ of 1,4-dioxane as a solvent. The reaction temperature and reaction time were set to be constant at $150{ }^{\circ} \mathrm{C}$ and $2 \mathrm{~h}$, respectively. Reaction products were analysed by gas chromatography using a "Chromatec crystal-2000M" GC (SKB Chromatec, Yoshkar-Ola, Russia) equipped with an FID detector and SE-54 column $(50 \mathrm{~m} \times 0.25 \mathrm{~mm})$. All catalytic tests were carried out three times or more to estimate the degree of errors and experimental reproducibility. The degree of errors for catalytic tests does not exceed 3\% rel. Formaldehyde conversion $(X)$, selectivity toward products $\left(S_{i}\right)$ and activity $(\mathrm{A})$ were average meaning and calculated according to following equations as:

$$
\begin{gathered}
X=\frac{\text { moles of } \mathrm{CH}_{2} \mathrm{O} \text { converted into all products }}{\text { moles of } \mathrm{CH}_{2} \mathrm{O} \text { loaded }} \times 100 \% \\
S_{i}=\frac{\text { moles of } \mathrm{CH}_{2} \mathrm{O} \text { converted into } i \text { - product }}{\text { moles of } \mathrm{CH}_{2} \mathrm{O} \text { converted into all products }} \times 100 \% \\
\mathrm{~A}=\frac{\text { moles of } \mathrm{CH}_{2} \mathrm{O} \text { converted into all products }}{\text { acid site number }}
\end{gathered}
$$

For the stability estimation spent catalyst was separated from the liquid product by the paper filter, then dried at $110^{\circ} \mathrm{C}$ overnight to remove 1,4-dioxane and unreacted formaldehyde. Recovered catalyst (more than $85 \%$ of initial loading) was mixed with a fresh one to keep a constant mass of the sample. Regenerated samples were obtained by calcination in air at $600{ }^{\circ} \mathrm{C}$ overnight.

According to $[28,29]$, it is necessary to carry out the deactivation tests under the kinetic-controlled conditions of the reaction, i.e., at the low or the medium conversion level, thus all deactivation tests were carried out at the conversion below $50 \%$.

The Equation (4), that is the kinetic equation of the reaction in the batch-type reactor, was chosen to describe the reaction performance. It had been proposed that the deactivation can be described as a zero order, first order or second order reaction, thus function $A$ vs. $t_{R}, \ln (A)$ vs. $t_{R}$ and $A^{-1}$ vs. $t_{R}$ were plotted and analysed $[30,31]$. The second-order equation (5), which is a function of the residence time of the catalyst in the reaction mass $\left(t_{R}\right)$, was taken for the deactivation model estimation, since the reactions of the formaldehyde conversion are the bimolecular and the deactivation order was confirmed from the experimental data analysis (Figure 3, Figures S7 and S8). The Equation (6) for the activity vs. time function was obtained by solving Equations (4) and (5) together and applied for 
the experimental data description. The equation parameters $\mathrm{A}^{0}$ and $t_{R}$ had been obtained via the regression analysis method.

$$
\begin{gathered}
\frac{d c}{d t}=-k_{R} \times c \times \theta(t) \\
\frac{d \theta}{d t}=-k_{D} \times \theta^{2} \\
\mathrm{~A}=\frac{\mathrm{A}^{0}}{\left(1+k \times \mathrm{t}_{R}\right)}
\end{gathered}
$$

\section{Conclusions}

In the present study, the liquid-phase Prins reaction between propene and formaldehyde was studied over H-BEA, H-FAU, H-MFI and H-MOR zeolites. It was found that the $\mathrm{H}$-BEA is the most active (formaldehyde conversion $45.1 \%$ ) and most selective catalyst for buta-1,3-diene production (10.0\%); the H-FAU can be used for 4-methyl-1,3-dioxane synthesis (selectivity $32.5 \%$ ), while the H-MFI is the most suitable catalyst for 3-buten-1-ol production. The zeolite samples were characterized by TPD- $\mathrm{NH}_{3}$ and nitrogen physisorption methods to estimate how acidic and textural properties influence activity and selectivity. According to obtained data, the acidity influences catalyst activity, while textural properties control product distribution, which is in agreement with previous reports $[13,14,23]$.

Spend samples had been studied by the TGA/DSC/DTA technique to estimate differences in catalyst deactivation by coke. The broad-pores samples (H-FAU and H-BEA) had more coke disposition than H-MOR and H-MFI, which are similar in terms of pore volume and effective pore diameter values. In addition, stability tests were carried out for the H-BEA and H-MFI samples; the catalysts were recycled after the reaction without calcination. It was found that the activity of both samples fell down cycle by cycle, while selectivity values toward buta-1,3-diene and 3-buten-1-ol increased. It was established that both samples deactivation processes are of the second order, but the deactivation rates were not equal; the H-BEA deactivation was slower than the H-MFI. These differences in deactivation are clearly related to the acidic and textural properties of the samples; higher strong acid number leads to higher deactivation rate, while the structural properties controlled the acid site accessibility.

Supplementary Materials: The following are available online at https:/ /www.mdpi.com/article/10 .3390/catal11101181/s1, Figure S1: XRD patterns, Figure S2: The nitrogen physisorption isotherms, Figure S3: The Horwath-Kawazoe pore distribution, Figure S4: The TCD-NH $\mathrm{N}_{3}$ curves, Figure S5: The TGA curves of spend samples, Figure S6: The DSC curves of spend samples, Figure S7: Activity vs. $t_{R}(A)$ and $\ln \left(\right.$ activity) vs. $t_{R}$ (B) curves for H-BEA, Figure S8: Activity vs. $t_{R}(A)$ and $\ln$ (activity) vs. $t_{R}(B)$ curves for H-MFI, Figure S9: The calculated $X$ vs. experimental $X$ for H-BEA, Figure S9: The calculated $X$ vs. experimental $X$ for H-MFI, Table S1: XRF results, Table S2: Activity of regenerated samples.

Author Contributions: Conceptualization, S.P.B. and V.F.T.; methodology, S.P.B. and K.I.D.; investigation, S.P.B.; writing — original draft preparation, S.P.B.; writing—review and editing, K.I.D.; supervision, K.I.D.; project administration, K.I.D. and V.F.T. All authors have read and agreed to the published version of the manuscript.

Funding: This work was carried out within the State program of TIPS RAS.

Acknowledgments: The work was performed using the equipment of the Shared-Use Centre "Analytical Centre for Problems of Deep Oil Refining and Petroleum Chemistry" and "New petrochemical process, polymers and adhesives" at the TIPS RAS.

Conflicts of Interest: The authors declare no conflict of interest. 


\section{References}

1. Markets and Markets. Butadiene Market Worth 14,536.1 Kilotons by 2018. Available online: https:/ / www.marketsandmarkets. com/PressReleases/butadiene.asp (accessed on 11 October 2020).

2. Weissermel, K.; Arpe, H.-J. Industrial Organic Chemistry, 3rd ed.; VCH Publishers: New York, NY, USA, 1997; 481p.

3. Statista. Market Volume of Isoprene in the United States from 2014 to 2025. Available online: https:/ /www.statista.com/statistics/ 799009/us-isoprene-market-volume/ (accessed on 11 October 2020).

4. Torres Galvis, H.M.; De Jong, K.P. Catalysts for production of lower olefins from synthesis gas: A review. ACS Catal. 2013, 3, 2130-2149. [CrossRef]

5. Khadzhiev, S.N.; Magomedova, M.V.; Peresypkina, E.G. Mechanism of olefin synthesis from methanol and dimethyl ether over zeolite catalysts: A review. Pet. Chem. 2014, 54, 245-269. [CrossRef]

6. Khadzhiev, S.N.; Dement'ev, K.I.; Gerzeliev, I.M. Catalytic cracking of alternative raw materials and their mixtures with petroleum fractions over microspherical zeolite-containing catalysts. Pet. Chem. 2014, 54, 1-9. [CrossRef]

7. Palankoev, T.A.; Dement'ev, K.I.; Kuznetsova, D.V.; Bondarenko, G.N.; Maximov, A.L. Acetone Reaction Pathways as a Model Bio-oxygenate in a hydrocarbon medium on zeolite Y and ZSM-5 catalysts: In Situ FTIR Study. ACS Sustain. Chem. Eng. 2020, 8, 10892-10899. [CrossRef]

8. Bedenko, S.P.; Dement'ev, K.I.; Tret'yakov, V.F.; Maksimov, A.L. The Prins reaction over heterogeneous catalysts (a Review). Pet. Chem. 2020, 60, 723-730. [CrossRef]

9. Dumitriu, E.; Gongescu, D.; Hulea, V. Contribution to the study of isobutene condensation with formaldehyde catalyzed by zeolites. Stud. Surf. Sci. Catal. 1993, 78, 669-676.

10. Dumitriu, E.; Hulea, V.; Hulea, T.; Chelaru, C.; Kaliaguine, S. Selective synthesis of isoprene by Prins condensation using molecular sieves. Stud. Surf. Sci. Catal. 1994, 84, 1997-2004.

11. Dumitriu, E.; Trong On, D.; Kaliaguine, S. Isoprene by Prins condensation over acidic molecular sieves. J. Catal. 1997, 170, 150-160. [CrossRef]

12. Dumitriu, E.; Hulea, V.; Fechete, I.; Catrinescu, C.; Auroux, A.; Lacaze, J.-F.; Guimon, C. Prins condensation of isobutylene and formaldehyde over Fe-silicates of MFI structure. Appl. Catal. A Gen. 1999, 181, 15-28. [CrossRef]

13. Vasiliadou, E.S.; Li, S.; Caratzoulas, S.; Lobo, R.F. Formaldehyde-isobutene Prins condensation over MFI-type zeolites. Catal. Sci. Technol. R. Soc. Chem. 2018, 8, 5794-5806. [CrossRef]

14. Vasiliadou, E.S.; Gould, N.S.; Lobo, R.F. Zeolite-catalyzed formaldehyde-propylene Prins condensation. ChemCatChem 2017, 9 , 4417-4425. [CrossRef]

15. Sangthong, W.; Probst, M.; Limtrakul, J. Computational study of the carbonyl-ene reaction of encapsulated formaldehyde in Na-FAU zeolite. J. Mol. Struct. 2005, 748, 119-127. [CrossRef]

16. Fu, H.; Xie, S.; Fu, A.; Ye, T. Theoretical study of the carbonyl-ene reaction between formaldehyde and propylene on the MgY zeolite. Comput. Theor. Chem. 2012, 982, 51-57. [CrossRef]

17. Wannakao, S.; Khongpracha, P.; Limtrakul, J. Density functional theory study of the carbonyl-ene reaction of encapsulated formaldehyde in $\mathrm{Cu}(\mathrm{I}), \mathrm{Ag}(\mathrm{I})$, and Au(I) exchanged FAU zeolites. J. Phys. Chem. A 2011, 115, 12486-12492. [CrossRef]

18. Hölderich, W.; Hesse, M.; Näumann, F. Zeolites: Catalysts for organic syntheses. Angew. Chem. Int. Ed. Engl. 1988, 27, 226-246. [CrossRef]

19. Guisnet, M.; Magnoux, P. Fundamental description of deactivation and regeneration of acid zeolites. Stud. Surf. Sci. Catal. 1994, $88,53-68$.

20. Magnoux, P.; Rabeharitsara, A.; Cerqueira, H.S. Influence of reaction temperature and crystallite size on HBEA zeolite deactivation by coke. Appl. Catal. A Gen. 2006, 304, 142-151. [CrossRef]

21. Bjørgen, M.; Olsbye, U.; Kolboe, S. Coke precursor formation and zeolite deactivation: Mechanistic insights from hexamethylbenzene conversion. J. Catal. 2003, 215, 30-44. [CrossRef]

22. Yan, T.; Yang, L.; Dai, W.; Wang, C.; Wu, G.; Guan, N.; Hunger, M.; Li, L. On the deactivation mechanism of zeolite catalyst in ethanol to butadiene conversion. J. Catal. 2018, 367, 7-15. [CrossRef]

23. Bedenko, S.P.; Kozhevnikov, A.A.; Dement'ev, K.I.; Tret'yakov, V.F.; Maximov, A.L. The Prins condensation between $i$-butene and formaldehyde over modified BEA and MFI zeolites in liquid phase. Catal. Commun. 2020, 138, 105965. [CrossRef]

24. Arundale, E.; Mikeska, L.A. The olefin-aldehyde condensation: The Prins reaction. Chem. Rev. 1952, 51, 505-555. [CrossRef]

25. Flego, C.; Galasso, L.; Kiricsi, I.; Clericiet, M.G. TG-DSC, UV-VIS-IR Studies on catalysts deactivated in alkylation of isobutane with 1-butene. Stud. Surf. Sci. Catal. 1994, 88, 585-590.

26. Wojciechowski, B.W. The kinetic foundations and the practical application of the time on stream theory of catalyst decay. Catal. Rev. 1974, 9, 79-113. [CrossRef]

27. Smit, B.; Maesen, T.L.M. Towards a molecular understanding of shape selectivity. Nature 2008, 451, 671-678. [CrossRef]

28. Schüth, F.; Ward, M.D.; Buriak, J.M. Common pitfalls of catalysis manuscripts submitted to Chemistry of materials. Chem. Mater. 2018, 30, 3599-3600. [CrossRef]

29. Scott, S.L. A matter of life(time) and death. ACS Catal. 2018, 8, 8597-8599. [CrossRef] 
30. Gayubo, A.G.; Aguayo, A.T.; Olazar, M.; Vivanco, R.; Bilbao, J. Kinetics of the irreversible deactivation of the HZSM-5 catalyst in the MTO process. Chem. Eng. Sci. 2003, 58, 5239-5249. [CrossRef]

31. Janssens, T.V.W. A new approach to the modeling of deactivation in the conversion of methanol on zeolite catalysts. J. Catal. 2009, 264, 130-137. [CrossRef] 